\title{
Caracterización morfoestructural e índices zoométricos de hembras Ovino de Pelo Criollo Colombiano "OPC" Sudán
}

\author{
Julio Flórez $\mathrm{M}^{1 *} \otimes(\mathbb{0})$ M.Sc; Marcos Hernández $\mathrm{P}^{1} \otimes(\mathbb{0}) \mathrm{MVZ}$; \\ Moris Bustamante $Y^{1} \bowtie(\mathbb{0})$ M.Sc; Oscar Vergara G ${ }^{1} \otimes$ Ph.D.
}

1Universidad de Córdoba, Facultad de Medicina Veterinaria y Zootecnia, Departamento de Ciencias Pecuarias, Grupo de Investigación en Producción Animal Tropical (GIPAT), Montería, Colombia.*Correspondencia: juliomateo7@hotmail.com

Recibido: Abril 2019; Aceptado: Febrero 2020; Publicado: Agosto 2020.

\section{RESUMEN}

Objetivo. El objetivo de este estudio fue describir las medidas corporales e índices zoométricos de Ovinos de Pelo Criollos Colombianos (OPC), en dos de sus variedades, Sudán Bayo y Sudán Blanco. Materiales y métodos. Esta investigación fue llevada a cabo en los departamentos de Córdoba, Cesar y La Guajira, se visitaron dos granjas en cada uno de los primeros dos departamentos y una en el tercero. 21, 75 y 14 animales por departamento fueron evaluados, respectivamente. Se estudiaron 29 variables morfoestructurales y el peso corporal en 110 ovejas Sudán, y con estas variables evaluadas, se construyeron 15 índices zoométricos. A la información recolectada se le aplicó estadística descriptiva mediante el software InfoStat ${ }^{\circledR}$. Resultados. Las Sudán Bayo presentaron valores superiores a las Sudán Blanco para el peso (40.8 y $40.57 \mathrm{~kg}$ ), al igual que para todas las medidas zoométricas relativas a la cabeza, las extremidades y la mayoría de las del tronco; para la primera variedad se emplearon 83 ejemplares y para la segunda 27. En cuanto a los índices zoométricos, las Sudán se caracterizaron por ser dolicocraniotas, dolicocéfalas, eumétricas, de tórax con tendencia elíptica, de grupas convexilíneas y de acuerdo a dos índices etnológicos longilíneas, mientras que con base en un índice funcional brevilíneas. Conclusiones. A través de esta investigación se conoció de manera detallada el estado morfométrico actual de las OPC Sudán, así como también la proporcionalidad entre las regiones corporales de estas, cuya inclinación es hacia el biotipo cárnico. Esta información es de importancia para el conocimiento, caracterización racial, definición de biotipo, conservación, fomento y mejora genética de los OPC.

Palabras clave: Morfología; morfometría; oveja; razas nativas; recursos genéticos (Fuentes: National agricultural library, AGROVOC).

\section{ABSTRACT}

Objective. The objective of this study was to describe the body measurements and zoometric indices of Sudán Bayo and Sudán Blanco, two breed varieties of Colombian creole woolless sheep (OPC). Materials and methods. This research was carried out in the departments of Córdoba, Cesar, and 
La Guajira. Two farms were visited in each of the first two departments and one farm in the third department. Twenty-one, 75, and 14 animals were evaluated in each department, respectively. Twenty-nine morphostructural variables and body weight were studied in 110 Sudán sheep. Based on this, 15 zoometric indices were also estimated. Descriptive statistic was applied to the information collected through the InfoStat ${ }^{\circledR}$ software. Results. Sudán Bayo sheep had higher values than Sudán Blanco sheep for the body weight $(40.8$ and $40.57 \mathrm{~kg})$, as well as for all the zoometric measurements relative to the head and extremities, and most of the trunk morphometric measurements; in the first breed variety 83 ewes were used and in the second 27 individuals. Regarding the zoometric indices, Sudán sheep were characterized as dolichocranial, dolichocephalic, eumetric, of thorax with elliptical tendency, convex rump, and longilineal animals according to two ethnological indices while brevilineal animals based on one functional index. Conclusions. Through this investigation, the current morphometric status of Sudán OPC sheep was known in detail, as well as the proportionality between the corporal regions of these, whose inclination is towards the meat biotype. This information is of importance for the breed recognition and characterization, biotype definition, conservation, development, and genetic improvement of OPC sheep.

Keywords: Morphology; morphometry; ewe, landraces; animal genetic resources (Sources: National agricultural library, AGROVOC).

\section{INTRODUCCIÓN}

Los Ovinos de Pelo Criollos Colombianos (OPC) Sudán, son básicamente el resultado de los ovinos de pelo llevados a las islas del Caribe desde las Islas Canarias (1), y en menor cuantía, de ovinos de África continental (2); estos grupos se cruzaron y sufrieron un largo periodo de selección natural, produciendo como resultado la formación de diferentes poblaciones, de las cuales destacan en Colombia los Sudán y Etíope, ambas muy bien adaptadas al trópico bajo. Los OPC Sudán desarrollaron una muy buena adaptación a las condiciones del trópico bajo colombiano (2), proveyendo así de seguridad agroalimentaria a los campesinos. Además, ha expresado un marcado potencial cárnico, si se considera que las condiciones bajo las cuales ha operado tal producción de carne se alejan bastante de las estimadas como óptimas. Igualmente, se han caracterizado también por ser de relevancia en actos sociales y culturales.

Los Sudán se han visto amenazados entre el 2005 y actualmente el 2020, dado al crecimiento de la producción ovina en Colombia desde principios del siglo XXI y al desconocimiento sobre la importancia de los OPC, factores los cuales han permitido que se popularice en los sistemas productivos que utilizan ovinos criollos, la introducción y cruzamiento indiscriminado con razas foráneas que son mejoradas para la producción de carne en otras condiciones ambientales, pero no para las características de adaptación como lo son la resistencia a enfermedades y las características reproductivas.

La situación expuesta es grave, ya que no es clara la delimitación de los OPC como raza. A pesar que desde la genética (2) y la morfometría (3) se ha sugerido que se cataloguen a los Sudán y Etíope como poblaciones racialmente diferentes, también existen estudios desde ambos ámbitos en los cuales no existe la discriminación entre estas clases de OPC $(4,5,6,7)$, reflejando ello que no hay un criterio unificado en cuanto a si los Sudán y Etíope son una raza con dos subrazas, o si son dos razas diferentes.

Para dar inicio a la conservación y al fomento de los Sudán, una de las actividades que se debe realizar de manera inicial es definir su clasificación como raza o subraza, siendo necesario para ello previamente realizar una caracterización morfológica cuantitativa, la cual está compuesta por la caracterización morfoestructural y la estimación de los índices zoométricos (8), los cuales se implementaron en este estudio con el objetivo de conocer el estado actual de estos OPC en específico, sus dimensiones, proporciones corporales e inclinación productiva. De tal manera, que posteriormente se contribuya a la creación de un estándar racial y a un conocimiento más objetivo del potencial zootécnico con el que estos ovinos cuentan, información con la que finalmente se aportará al reconocimiento racial de los Sudán y su mejoramiento genético. 


\section{MATERIALES Y MÉTODOS}

Tipo de estudio. Este estudio fue descriptivo, de corte transversal, y el muestreo no probabilístico por conveniencia.

Localización. Las ovejas Sudán Bayo se localizaron en los municipios de Valledupar (Cesar; 10007'37.3" LN - 73039'45.4" LO y $10^{\circ} 07^{\prime} 01.4^{\prime \prime}$ LN - 73033'02.2" LO), Montería y Ciénaga de Oro (Córdoba; 8034'15.7" LN 75056'54.5" LO y 8052'43.1" LN - 75042'12.3" LO). Respecto a las ejemplares Sudán Blanco, estas se encontraron ubicadas en los municipios de San Juan del Cesar (La Guajira; 10049'46.0" LN - 72056'53.8" LO) y Valledupar (Cesar; 10025'36.6" LN - 73021'16.0" LO). Los lugares de La Guajira y Cesar recién mencionados se consideran como Bosque Muy Seco Tropical, mientras que para el caso de Córdoba Bosque Seco Tropical.

Animales de estudio. LOS OPC Sudán son descritos inicialmente desde lo faneróptico en 1977, expresándose principalmente que poseen un color de capa amarillo (Figura 1 ) que en ocasiones puede ser blanco (Figura 2), perfil «rectilíneo semiconvexo», presentan ausencia de cuernos, orejas direccionadas horizontalmente, cortas y pezuñas de pigmentación oscuras o claras (2). Cabe destacar que las Sudán Blanco son marcadamente reducidas si se contrastan con las Sudán Bayo, en términos de tamaño poblacional.

Aunque no existe un consenso sobre si los Sudán y Etíope son una raza, dos subrazas o dos razas, los estudios de Vivas (2) y Flórez et al (3) sugieren que estos dos recursos zoogenéticos que conforman al grupo OPC, pueden ser considerados como distintos racialmente.

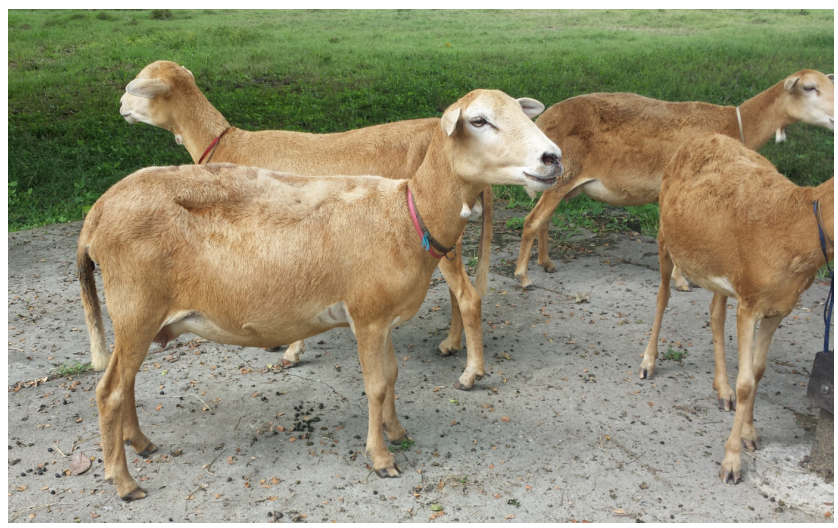

Figura 1. Ejemplares Sudán Bayo.

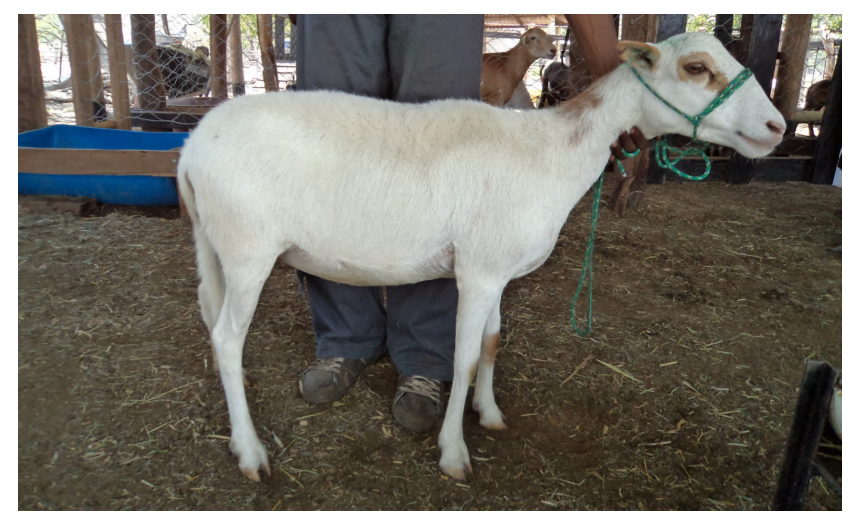

Figura 2. Ejemplar Sudán Blanco.

Manejo animal y características evaluadas. 87 hembras Sudán Bayo y 23 Sudán Blanco fueron evaluadas, teniendo que haber cumplido para ser escogidas: con la descripción exterior de OPC Sudán encontrada en la literatura (2), una edad no inferior a los dos años, un periodo de ayuno superior a 12 horas, carencia de anomalías anatómicas, así como también de mestizaje y gravidez evidente.

Para ejecutar la evaluación morfológica cuantitativa de las OPC Sudán se tuvo en cuenta lo reportado por Popoola (9), Morales-de la Nuez (10) y Bravo y Sepúlveda (11) tanto en variables morfoestructurales como en índices zoométricos. Las características evaluadas fueron:

Variables morfoestructurales. LCB, longitud de la cabeza; PCB, profundidad de la cabeza; LCN, longitud del cráneo; LCR, longitud de la cara; ACB, ancho de la cabeza; ACN, ancho del cráneo; TOR, tamaño de la oreja; TOJ, tamaño del ojo; $A C R$, alzada a la cruz; AD, alzada al dorso; AES, alzada al esternón; AG, alzada a la grupa; ANC, alzada al nacimiento de la cola; LCP, longitud corporal; DDE, diámetro dorsoesternal; AEN, ancho entre encuentros; DB, diámetro bicostal; AIL, ancho entre iliones; AIQ, ancho entre isquiones; LG, longitud de la grupa; PT, perímetro torácico; LCO, longitud de la cola; $\mathrm{PR}$, perímetro de la rodilla; $\mathrm{PMC}$, perímetro del metacarpo; PMT, perímetro del metatarso; PME, perímetro del menudillo; PCU, perímetro de la cuartilla; PCO, perímetro de la corona; y PCV, perímetro del corvejón. A este grupo se añadió el PC, peso corporal, debido a su importancia productiva ya que tiene alta relación con las variables morfoestructurales.

Índices zoométricos etnológicos. ICR, índice craneal $(=(A C N / L C N) * 100) ; \mathrm{ICE}$, índice cefálico $(=(A C B / L C B) * 100) ; \mathrm{ICL}$, índice corporal 
$(=(\mathrm{LCP} / \mathrm{PT}) * 100) ; \mathrm{ICM}$, índice de compacidad $(=(\mathrm{PC} / \mathrm{ACR}) * 100) ; \mathrm{ITO}$, índice torácico $(=(\mathrm{DB} /$ $\mathrm{DDE}) * 100) ;$ IDR, índice alzada de dorso y cruz $(=(A D / A C R) * 100) ;$ IDP, índice alzada de dorso y pelvis $(=(A D / A G) * 100)$; e IPV, índice pélvico $(=(\mathrm{AIL} / \mathrm{LG}) * 100)$

Índices zoométricos funcionales. IPR, índice de profundidad relativa del tórax (=(DDE/ ACR $* 100) ; \mathrm{ICO}$, índice de cortedad relativa $(=(A C R / L C P) * 100) ;$ IPT, índice pelviano transversal $(=(A I L / A C R) * 100) ; I P L$, índice pelviano longitudinal $(=(\mathrm{LG} / \mathrm{ACR}) * 100)$; IER, índice de espesor relativo del metacarpo $(=(P M C)$ ACR $* 100)$; IMT, índice metacarpo-torácico $(=(\mathrm{PMC} / \mathrm{PT}) * 100) ;$ e IDO, índice dáctilocostal $(=(P M C / D B) * 100)$.

Las variables cefálicas se midieron mediante un compás de espesores, a excepción del ACN, TOR y TOJ, con los que se utilizó un pie de rey. En las variables del tronco, las alzadas se tomaron con un bastón zoométrico, el PT, la LCO y la LCP se midieron con una cinta métrica, mientras que el resto de características de esta región se evaluaron con un compás de espesores. Todas las medidas de las extremidades se tomaron con cinta métrica. Para el PC se empleó una báscula portátil.

Análisis de los resultados. A las variables morfoestructurales y los índices zoométricos una vez estimados, se les aplicó estadística descriptiva, más específicamente, media, desviación estándar y valores tanto mínimos como máximos. Esto se realizó a través del software InfoStat ${ }^{\circledR}$ versión 2016I (12).

\section{RESULTADOS}

Caracterización morfoestructural. En la tabla 1 están descritos los estadísticos relativos a las 30 variables evaluadas. Como primera característica se tiene el PC, el cual en las Sudán Blanco fue menor que en las Sudán Bayo por una diferencia de $0.23 \mathrm{~kg}$, siendo el PC promedio de las primeras $40.57 \mathrm{~kg}$. En las variables morfoestructurales de la región cefálica, se observó que las hembras Sudán Bayo siempre presentaron medias superiores a las de las Sudán Blanco.

Las Sudán Blanco manifestaron un mayor valor promedio para las alzadas que las Sudán Bayo, aunque de forma leve; fue excepción a esto la ANC. La situación mencionada inicialmente en este párrafo se repitió para la AEN, AIQ y LCO. Respecto al resto de las variables zoométricas del tronco, las Sudán Bayo tuvieron medias superiores a las Sudán Blanco, estas medidas están relacionas con el largo y ancho del cuerpo y el tamaño de la grupa. En las circunferencias evaluadas en las extremidades, las OPC Sudán Bayo presentaron mayores cifras que las Sudán Blanco.

Índices zoométricos. Con el fin de determinar la proporcionalidad corporal de las OPC, y a su vez la inclinación productiva, se estimó el grado de relación entre algunas variables de las diferentes regiones corporales, a través del cálculo de ocho índices etnológicos y siete funcionales. La estadística descriptiva para dichos índices zoométricos puede ser encontrada en la tabla 2.

El primer índice etnológico estudiado fue el ICR, de acuerdo con el cual las Sudán poseen un aspecto dolicocraniota, porque predomina el largo del cráneo frente a la amplitud de este, siendo válida la misma analogía para el ICE, pero teniendo en cuenta la LCB y el ACB, índice el cual cataloga a las Sudán como dolicocéfalas.

Con base en el ICM, se determinó que el formato de esta población es eumétrico. En función a los valores obtenidos a través de los análisis del ITO, las Sudán se clasifican como de tórax de tendencia elíptica y longilíneas. De igual manera, con base en el ICL las Sudán se catalogaron como longilíneas, ya que las medias fueron superiores a 88 .

A través del IDR, se observó que las Sudán Bayo en promedio suelen presentar una leve depresión a nivel dorsal. Respecto al IDP, tanto en Sudán Bayo como en Sudán Blanco la grupa presenta una mayor alzada que el dorso, pero poseen mayor horizontalidad de la espalda las últimas mencionadas. Como último índice etnológico se calculó el IPV, mediante el cual se obtuvo que las Sudán presentan una grupa convexilínea.

Pasando a los índices funcionales, el IPR señaló a las Sudán Bayo como más profundas de tórax respecto al largo de sus patas, si se les compara con las Sudán Blanco. De acuerdo con el ICO, las Sudán se clasifican como brevilíneas. Los valores promedios para el IPT en las Sudán estuvieron por debajo, pero no muy lejanos, a lo considerado como óptimo para la producción de carne, mientras que para el IPL presentaron cifras deseables en animales de aptitud cárnica. Acerca el IER, este mostró valores regulares para la producción de carne. 
Tabla 1. Estadísticos descriptivos de las variables morfoestructurales en hembras OPC Sudán Bayo y Sudán Blanco.

\begin{tabular}{|c|c|c|c|c|c|}
\hline Variables morfoestructurales $(\mathrm{cm})$ & Ovejas Sudán & Media & DE & Máx. & Mín. \\
\hline \multirow{2}{*}{ Peso corporal* } & Bayo & 40.8 & 5.71 & 58 & 27.5 \\
\hline & Blanco & 40.57 & 8.13 & 56.5 & 27 \\
\hline \multirow{2}{*}{ Longitud de la cabeza } & Bayo & 20.4 & 1.05 & 23.1 & 16.4 \\
\hline & Blanco & 20 & 0.86 & 22.2 & 18.5 \\
\hline \multirow{2}{*}{ Profundidad de la cabeza } & Bayo & 14.59 & 0.72 & 16.3 & 13 \\
\hline & Blanco & 14.23 & 0.87 & 15.4 & 12.55 \\
\hline \multirow{2}{*}{ Longitud del cráneo } & Bayo & 12.1 & 1.1 & 16.25 & 9.5 \\
\hline & Blanco & 10.81 & 0.92 & 13.35 & 9.8 \\
\hline \multirow{2}{*}{ Longitud de la cara } & Bayo & 15.92 & 1.06 & 19 & 12.5 \\
\hline & Blanco & 15.49 & 0.98 & 17.45 & 13.25 \\
\hline \multirow{2}{*}{ Ancho de la cabeza } & Bayo & 11.87 & 1.06 & 19.95 & 10.35 \\
\hline & Blanco & 8.46 & 1.07 & 12.05 & 7.45 \\
\hline \multirow{2}{*}{ Ancho del cráneo } & Bayo & 8.55 & 0.34 & 9.25 & 7.6 \\
\hline & Blanco & 8.22 & 0.44 & 9.35 & 7.45 \\
\hline \multirow{2}{*}{ Tamaño de la oreja } & Bayo & 11.79 & 0.87 & 14.1 & 9.55 \\
\hline & Blanco & 11.68 & 1.01 & 13.75 & 9.95 \\
\hline \multirow{2}{*}{ Tamaño del ojo } & Bayo & 2.83 & 0.21 & 3.4 & 2.4 \\
\hline & Blanco & 2.7 & 0.24 & 3.1 & 2.2 \\
\hline \multirow{2}{*}{ Alzada a la cruz } & Bayo & 67.21 & 2.95 & 77.7 & 59.1 \\
\hline & Blanco & 67.72 & 4.32 & 76.7 & 60.1 \\
\hline \multirow{2}{*}{ Alzada al dorso } & Bayo & 67.14 & 2.86 & 74.9 & 59.6 \\
\hline & Blanco & 68.07 & 4.15 & 76 & 59.7 \\
\hline \multirow{2}{*}{ Alzada al esternón } & Bayo & 41.22 & 3.66 & 58.2 & 31.4 \\
\hline & Blanco & 41.35 & 3.5 & 49 & 35.7 \\
\hline \multirow{2}{*}{ Alzada a la grupa } & Bayo & 68.29 & 2.68 & 77.2 & 62.5 \\
\hline & Blanco & 68.54 & 3.52 & 74.7 & 61.3 \\
\hline \multirow{2}{*}{ Alzada al nacimiento de la cola } & Bayo & 60.37 & 3.19 & 71.6 & 54.4 \\
\hline & Blanco & 59.59 & 4.36 & 69.5 & 50.1 \\
\hline \multirow{2}{*}{ Longitud corporal } & Bayo & 74.73 & 4.07 & 86 & 63.3 \\
\hline & Blanco & 72.36 & 4.98 & 82.5 & 63 \\
\hline \multirow{2}{*}{ Diámetro dorsoesternal } & Bayo & 28.29 & 1.57 & 32 & 25 \\
\hline & Blanco & 28.16 & 2.62 & 33.4 & 23.2 \\
\hline & Bayo & 14.84 & 1.32 & 17.65 & 10 \\
\hline Ancho entre encuentros & Blanco & 14.94 & 2.11 & 18.4 & 10.2 \\
\hline & Bayo & 17.59 & 1.74 & 21.8 & 14.1 \\
\hline Diametro bicostal & Blanco & 17 & 2.88 & 21.6 & 10.8 \\
\hline & Bayo & 16.52 & 1.25 & 19.9 & 13.8 \\
\hline Ancho entre Illones & Blanco & 15.44 & 1.75 & 19 & 12 \\
\hline & Bayo & 6.26 & 0.83 & 8.2 & 4.3 \\
\hline Ancho entre isquiones & Blanco & 6.32 & 0.69 & 8 & 5.1 \\
\hline & Bayo & 20.82 & 1.28 & 28 & 17.5 \\
\hline Longitud de la grupa & Blanco & 20.46 & 1.56 & 23 & 16.9 \\
\hline & Bayo & 80.71 & 4.67 & 94 & 65.7 \\
\hline Perımetro toracıco & Blanco & 80.62 & 8.48 & 97.4 & 64.2 \\
\hline & Bayo & 33.02 & 3.79 & 41.7 & 22.6 \\
\hline Longitud de la cola & Blanco & 33.28 & 6.7 & 43.5 & 16 \\
\hline & Bayo & 12.6 & 0.69 & 14 & 10.7 \\
\hline Perimetro de la rodilla & Blanco & 11.98 & 0.85 & 13.4 & 10.2 \\
\hline & Bayo & 7.46 & 0.43 & 8.75 & 6.1 \\
\hline Perımetro del metacarpo & Blanco & 7.19 & 0.57 & 8.2 & 5.9 \\
\hline & Bayo & 8.65 & 0.53 & 10.1 & 7.4 \\
\hline Perımetro del metatarso & Blanco & 8.37 & 0.71 & 9.4 & 6.9 \\
\hline & Bayo & 11.77 & 0.68 & 13.4 & 10.4 \\
\hline Perımetro del menudıllo & Blanco & 11.36 & 0.72 & 12.5 & 9.85 \\
\hline & Bayo & 10.28 & 0.66 & 11.8 & 8.9 \\
\hline Perımetro de la cuartılla & Blanco & 10.03 & 0.76 & 11.8 & 8.5 \\
\hline & Bayo & 15.61 & 0.98 & 18 & 13.2 \\
\hline Perimetro de la corona & Blanco & 15.5 & 1.1 & 17 & 13.4 \\
\hline & Bayo & 17.09 & 0.92 & 19 & 15 \\
\hline Perimetro del corvejon & Blanco & 16.55 & 1.28 & 18.7 & 13.6 \\
\hline
\end{tabular}

*Unidad de medida en kilogramos; DE = desviación estándar; Máx. = máximo; Mín. = mínimo. 
Tabla 2. Estadísticos descriptivos de los índices zoométricos en hembras OPC Sudán Bayo y Sudán Blanco.

\begin{tabular}{|c|c|c|c|c|c|c|}
\hline & Índices zoométricos & Ovejas Sudán & Media & DE & Máx. & Mín. \\
\hline \multirow{16}{*}{ 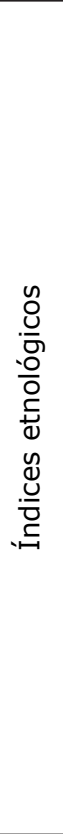 } & \multirow{2}{*}{ Índice craneal } & Bayo & 71.11 & 5.71 & 82.35 & 52.62 \\
\hline & & Blanco & 76.39 & 5.91 & 87.62 & 64.04 \\
\hline & \multirow{2}{*}{ Índice cefálico } & Bayo & 58.27 & 5.41 & 97.32 & 48.86 \\
\hline & & Blanco & 42.31 & 5.08 & 58.78 & 37.16 \\
\hline & \multirow{2}{*}{ Índice corporal } & Bayo & 92.67 & 5.53 & 115.68 & 81.88 \\
\hline & & Blanco & 90.27 & 6.66 & 107.03 & 83.24 \\
\hline & \multirow{2}{*}{ Índice de compacidad } & Bayo & 60.6 & 7.42 & 80.67 & 40.5 \\
\hline & & Blanco & 59.4 & 9.89 & 77.29 & 42.08 \\
\hline & \multirow{2}{*}{ Índice torácico } & Bayo & 62.18 & 5.11 & 73.61 & 52.78 \\
\hline & & Blanco & 60.16 & 7.19 & 73.97 & 44.81 \\
\hline & \multirow{2}{*}{ Índice alzada de dorso y cruz } & Bayo & 99.96 & 3.4 & 108.12 & 85.89 \\
\hline & & Blanco & 100.69 & 2.72 & 106.18 & 96.57 \\
\hline & \multirow{2}{*}{$\begin{array}{c}\text { Índice alzada de dorso y } \\
\text { pelvis }\end{array}$} & Bayo & 98.33 & 2.27 & 102.15 & 85.88 \\
\hline & & Blanco & 99.29 & 1.91 & 103.21 & 96.07 \\
\hline & \multirow{2}{*}{ Índice pélvico } & Bayo & 79.52 & 5.91 & 95 & 55 \\
\hline & & Blanco & 75.56 & 7.27 & 89.2 & 62.5 \\
\hline \multirow{14}{*}{ 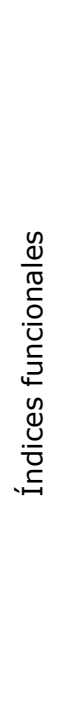 } & \multirow{2}{*}{$\begin{array}{l}\text { Índice de profundidad relativa } \\
\text { del tórax }\end{array}$} & Bayo & 42.11 & 2.14 & 47.14 & 37.11 \\
\hline & & Blanco & 41.59 & 2.4 & 45.79 & 36.15 \\
\hline & \multirow{2}{*}{ Índice de cortedad relativa } & Bayo & 90.15 & 5.43 & 107.27 & 75.29 \\
\hline & & Blanco & 93.5 & 4.49 & 103.23 & 87.1 \\
\hline & \multirow{2}{*}{ Índice pelviano transversal } & Bayo & 24.6 & 1.73 & 30.29 & 20.38 \\
\hline & & Blanco & 22.76 & 2.24 & 26.09 & 19.48 \\
\hline & \multirow{2}{*}{ Índice pelviano longitudinal } & Bayo & 31 & 1.91 & 41.24 & 25.07 \\
\hline & & Blanco & 30.25 & 1.64 & 33.48 & 26.87 \\
\hline & \multirow{2}{*}{$\begin{array}{c}\text { Índice de espesor relativo del } \\
\text { metacarpo }\end{array}$} & Bayo & 11.12 & 0.72 & 13.10 & 9.04 \\
\hline & & Blanco & 10.61 & 0.59 & 11.92 & 9.38 \\
\hline & \multirow{2}{*}{ Índice metacarpo-torácico } & Bayo & 9.26 & 0.7 & 12.18 & 7.82 \\
\hline & & Blanco & 8.95 & 0.45 & 9.63 & 8.11 \\
\hline & \multirow{2}{*}{ Índice dáctilocostal } & Bayo & 42.81 & 4.48 & 52.67 & 33.03 \\
\hline & & Blanco & 43.13 & 5.45 & 54.63 & 34.92 \\
\hline
\end{tabular}

$\mathrm{DE}$ = desviación estándar; Máx. = máximo; Mín. = mínimo.

Según el IMT las Sudán Blanco son, proporcionalmente hablando, más altas de patas y livianas que las Sudán Bayo de manera leve, pero bien proporcionadas en ambos casos; además, el IMT refleja una aparente buena funcionalidad lechera, al igual que también lo hace el IDO, siendo este último índice propiamente de aptitud lechera.

\section{DISCUSIÓN}

Caracterización morfoestructural. Respecto a lo obtenido para el PC en otras investigaciones realizadas con OPC, Arredondo et al (4) encontraron en hembras del Quindío y Valle del Cauca (34.4 $\mathrm{kg})$, valores que están por debajo al hallado en las Sudán evaluadas en el actual estudio. Cifras promedio superiores a las halladas en este trabajo para la LCB, han sido reportadas en OPC con $27.5 \mathrm{~cm}$ (4) y Pelibuey Mexicano con 21.88 cm (13), pudiéndose hacer la misma analogía para la ACN de las hembras Pelibuey Mexicano $(11.17 \mathrm{~cm})$, mientras que para la característica de LCN se observó lo contrario, pues las Pelibuey Mexicano presentaron un menor valor $(6.53 \mathrm{~cm})$ que las Sudán. Las variables morfoestructurales de la cabeza cobran importancia desde el punto 
de vista etnológico, debido a que estas son poco influenciables por las condiciones ambientales y es fundamental tenerlas en cuenta para la estandarización racial (14).

En cuanto a las variables morfoestructurales del tronco concernientes a las alzadas, Biagiotti et al (15) reportaron en hembras Santa Inés, valores superiores a los de las Sudán para la ACR (71.35 $\mathrm{cm})$ y la $A G(71.37 \mathrm{~cm})$. Mientras que medias de alzadas inferiores, fueron halladas en hembras de las razas: Pelibuey Mexicano $(A C R=65.18$ y $A G=64.55 \mathrm{~cm})$, Katahdin $(A C R=67.14$ y $A G=$ $66.62 \mathrm{~cm})$, Dorper $(A C R=65.14$ y $A G=64.98$ $\mathrm{cm})(16)$ y en hembras OPC de los departamentos de Sucre $(A E S=35.1, A D=63.6$ y $A N C=59.1$ $\mathrm{cm})(7)$, Quindío y Valle del Cauca $(A C R=63.3$ y $A G=63.6 \mathrm{~cm})(4)$.

Respecto a las variables morfométricas del tronco relativas a la grupa, fueron encontrados estudios con medias inferiores a las de la actual investigación para la LG, en hembras OPC $(19.9 \mathrm{~cm})(4)$, Pelibuey Mexicano $(19.2 \mathrm{~cm})$ (16) y Pelibuey Cubano $(20.27 \mathrm{~cm})(17)$. Medias superiores a las de la actual investigación para la AIL, fueron apreciadas en los estudios hechos con las razas Katahdin $(18.38 \mathrm{~cm})(16)$, Dorper (19.02 $\mathrm{cm})(16)$ y Pelibuey Mexicano $(17.63 \mathrm{~cm})(13)$.

En torno a las demás medidas zoométricas del tronco, el PT de las Sudán fue superior al de las OPC de Quindío y Valle del Cauca $(79,2 \mathrm{~cm})(4)$ e inferior, pero no muy distante, a lo reportado en OPC del Huila $(82.7 \mathrm{~cm})(6)$, Santa Inés $(86.39 \mathrm{~cm}$ (15) y Katahdin $(84.85 \mathrm{~cm})(16)$. Una situación similar a la anterior, fue encontrada para el DDE, AEN y DB, en ovejas Pelibuey Mexicano $(\mathrm{DDE}=31.16$ y $\mathrm{AEN}=16.98 \mathrm{~cm})(13)$ y Canaria $(D D E=33.77$ y $D B=25.23 \mathrm{~cm})(18)$.

Las variables morfométricas de la región del tronco son de importancia en la evaluación morfoestructural, porque es en estas donde se aplican criterios de selección para la producción cárnica, pues dichos rasgos condicionan la primera impresión de los individuos de producción de carne, que está basada en el grado de compacidad, en lo ampuloso del tronco, en la profundidad y lo arqueado de costillas, lo largo y ancho de la grupa, el grado de desarrollo muscular de esta última y el perfil de la nalga, aspectos que influyen desde el rendimiento en canal, hasta en la capacidad digestiva, respiratoria y facilidad reproductiva (14).

Respecto a las extremidades, cifras superiores a los de este estudio para el PMC, fueron encontradas en ejemplares Pelibuey Mexicano
$(8.74 \mathrm{~cm})$, Katahdin $(9.69 \mathrm{~cm})$, Dorper $(9.82$ $\mathrm{cm})(16)$ y Canaria $(8.01 \mathrm{~cm})(18)$. Por otro lado, en hembras OPC $(7.3 \mathrm{~cm})(4)$ y Black Belly $(7.4$ $\mathrm{cm}$ ) (19) fueron reportadas medias superiores a la de las Sudán Blanco, pero inferiores a la de las Sudán Bayo.

Herrera y Luque (14), exponen que a través del grosor del PMC se determina la tendencia productiva de una raza, ya que entre mayor sea el grosor del individuo, presentará un mayor peso y por ende existirá más tendencia hacia una aptitud sarcopoyética.

Índices zoométricos. Los índices etnológicos están relacionados con la clasificación racial de los animales y con base en las cifras obtenidas en este apartado, se tiene que:

Fue hallado para el ICE en hembras OPC de Sucre una media de 43.5 (7), cifra que está por encima de los resultados obtenidos en Sudán Blanco, pero por debajo a lo encontrado en Sudán Bayo. Mientras que en OPC de Quindío y Valle del Cauca el ICE fue considerablemente menor al de esta investigación (36.21) (4).

Promedio para el ITO que al igual que el de las Sudán indicó ser de ejemplares longilíneos (estirados, estrechos y alargados; $\leq 85$ ), fue el encontrado en ovejas Canaria (73.96) (18); mientras que ovejas criollas del oeste formoseño en Argentina, presentaron una media de 186.15 (20), valor que clasifica a dicha población como brevilínea (de individuos acortados, anchos y espesos; $\geq 89$ ). Contrario a la actual investigación, Arredondo et al (4) clasificaron a las OPC de su estudio como brevilíneas con base en el ICL (81.60), debido a que hallaron una media inferior a 86 para este índice.

Cabe resaltar que Arredondo et al (4) determinaron de manera objetiva, que el grado de horizontalidad de la espalda en los OPC daba lugar a una línea dorsal recta u horizontal. Sin embargo, lo hallado en dichas investigaciones no concuerda con lo determinado en este artículo, donde se evidenció una línea dorsolumbar ascendente hacia la grupa, lo cual es propio de animales con escasa selección como las OPC Sudán. Esta condición repercute indeseadamente en ejemplares de aptitud cárnica, debido a que las tuberosidades ilíacas externas se elevan y contribuyen a una mayor inclinación de la pelvis, produciendo así un acortamiento de los músculos semitendinosos y semimembranosos (14).

Valores inferiores a 100 para el IPV, son propios de grupas convexilíneas, lo que quiere decir que 
predomina el largo sobre la anchura, tal como sucedió con las Sudán evaluadas en este trabajo, siendo reportadas también bajo la misma condición, grupas de ovejas OPC (75.9) (7) y Araucanas (81.08) (11). Es de importancia el IPV, porque orienta sobre la estructura de la grupa y presenta relación con la aptitud reproductiva de la raza.

A través de los índices funcionales se diagnostica la aptitud productiva de una población animal, obteniéndose en este estudio que:

En el IPR, los valores obtenidos para las Sudán se hallaron por debajo de 50, mientras que es deseable para la producción de carne cifras superiores. Fue encontrado un promedio superior a 50 en la raza Canaria (53.2) (18), siendo inferior a dicho número pero superior a la media de las Sudán, lo determinado en OPC de Quindío y Valle del Cauca (46.16) (4). En ovejas criollas Araucanas, se halló un índice de 40.23 (11), inferior a todos los mencionados anteriormente.

A través del ICO las Sudán se pueden catalogar como brevilíneas, ya que presentan valores inferiores a 95, aunque estos resultados no son concordantes con los obtenidos en el ITO e ICL, debido al tipo de variables morfoestructurales usadas para estimar estos índices, los cuales son diferentes, por lo que la correcta interpretación de estos índices es: que a pesar de que en términos etnológicos las OPC Sudán poseen un tórax (ITO) que tiende a ser estirado, estrecho y alargado (longilíneo), así como también su tronco completamente (ICL), estos ejemplares desde lo funcional y en cuanto a la apariencia corporal de manera general (ICO) tienden a aproximarse más a un rectángulo, forma predominante en los animales de aptitud carnicera (brevilíneos).

Otro aspecto importante a resaltar, es que cuando los autores analizaron al ITO, ICL e ICO yendo más allá de la media aritmética de la población en general, encontraron que en las Sudán Bayo el 9.3 (ICL) u $88.51 \%$ (ICO) son brevilíneas, el 18.6 (ICL) o $10.34 \%$ (ICO) son mediolíneas, y el 100 (ITO), 72.09 (ICL) o 1.15\% (ICO) son longilíneas, apreciándose un comportamiento similar en las Sudán Blanco, donde el 39.13 (ICL) o $68.18 \%$ (ICO) fueron brevilíneas, el 21.74 (ICL) o $31.82 \%$ (ICO) mediolíneas, y el 100 (ITO) o 39.13\% (ICL) longilíneas. Aquí se aprecia que independientemente del índice estimado, es posible hallar en las OPC Sudán a través de un mismo índice, individuos que se pueden clasificar en diferentes categorías de la sistemática baroniana.
Por lo tanto, se recomienda que antes de llegar a conclusiones a partir del promedio general de una población, para clasificarla según la sistemática de Barón, primero se determine el porcentaje de los ejemplares que son longilíneos, brevilíneos y/o mediolíneos.

Moreno et al (7), Ángel y Ramírez (6) y Arredondo et al (4) coinciden en concluir que los OPC son de apariencia brevilínea. Los autores del presente estudio concuerdan con dicha conclusión en términos generales, en especial porque dicha condición brevilínea está determinada por un índice funcional, es decir, desde una perspectiva productiva.

Para el IPT, ovejas Canarias de España obtuvieron una media superior (28.28) (18) en comparación con las Sudán, al igual que las ovejas Araucanas de Chile (29.55) (11). Sin embargo, todas estas poblaciones poseen cifras inferiores al valor mínimo deseado para la producción de carne, por lo tanto, se deduce que en estas ovejas la AIL no alcanza a ser la tercera parte de la ACR.

Respecto al IPL, las Sudán presentaron valores que se ubican por debajo de 37 , considerándose deseable las cifras inferiores a dicho número para la producción de carne. Una media inferior fue encontrada en hembras OPC (IPL = 29.6) (7).

El IMT es un índice de aptitud motora y se encarga de indicar la relación entre el volumen corporal y la osamenta, relación que indica que las Sudán no tienen problemas de sobrecarga en los aplomos. Además, reafirma que estos OPC son de tendencia eumétrica, tal como lo concluyeron Moreno et al (7) en OPC; sin embargo, Ángel y Ramírez (6) y Arredondo et al (4) han clasificado a los OPC como elipométricos.

Por último, se tiene el IDO, que es un índice de aptitud lechera que relaciona la masa corporal con la fortaleza de las extremidades, donde cifras entre 40 y 45 indican una buena funcionalidad para esta aptitud, rango en el que se encuentran los resultados obtenidos por las Sudán. Se halló una media inferior al de las Sudán en ovejas Canarias (IDO = 31.59) (18).

Los autores no consideran que los resultados obtenidos en las Sudán indiquen una tendencia hacia la aptitud lactopoyética, sino que los índices involucrados con esta aptitud se estiman con base en el PMC, partiendo del referente que animales de delgado metacarpo son de tendencia a la producción de leche (14). A pesar de que es cierto que existe relación entre delgado PMC y la aptitud lactopoyética, las poblaciones criollas desarrollan cañas que no son gruesas 
por cuestiones de adaptabilidad, pudiendo arrojar así aparentes valores de aptitud lechera que no implican necesariamente una verdadera inclinación hacia dicha producción.

Las diferencias encontradas en las Sudán a nivel de las variables e índices, con respecto a las otras razas discutidas, se atribuyen esencialmente a la influencia de factores de manejo, climatológicos y genéticos, siendo el primero de repercusiones más a corto plazo, el segundo de una mayor pertinencia en procesos evolutivos, mientras que el último, hace referencia al componente genético de las otras razas, que es diferente al de las OPC e influenciado generalmente por planes de mejora animal, lo cual suele poner en ventaja a las razas foráneas, con respecto a su potencial morfoestructural. En cuanto a las diferencias encontradas en otras poblaciones de OPC, no fueron marcadas en su mayoría y se explican principalmente por el manejo y las condiciones medio ambientales, que aunque similares, fueron desiguales.

Es de destacar, que las Sudán han presentado resultados relativamente buenos para la producción de carne, si se les compara con otras razas de pelo de mayor difusión o mejoradas, reflejándose esto en que más del $40 \%$ de estas OPC, están por encima de la media encontrada para el PC en hembras Pelibuey Mexicano (41.13 $\mathrm{kg}$ ) (16) y en mayor porcentaje, por encima del promedio del PC de ovejas Pelibuey Cubano $(35.78 \mathrm{~kg})$ (17) y Black Belly $(35.4 \mathrm{~kg})(19)$.

Finalmente, se puede concluir con base en lo hallado, que las Sudán se caracterizan por ser: dolicocraniotas, dolicocéfalas, de grupas siempre convexilíneas, de una línea dorsolumbar ascendente hacia la grupa con depresión a nivel dorsal en parte de las Sudán Bayo, de volumen corporal armónico con respecto al sistema óseo, de extremidades medianas tanto para el grosor como para la longitud, de formato eumétrico y peso que está alrededor de los $40 \mathrm{~kg}$, por ser de mayor talla las Sudán Bayo que las Blanco, por ser ejemplares longilíneos desde lo etnológico, pero funcionalmente brevilíneos, así como también funcionalmente se caracterizan por presentar una tendencia moderada hacia la aptitud cárnica.

Lo expuesto con anterioridad es de importancia, puesto a que aparte de brindar una actualización del estado morfoestructural actual de las Sudán, es la primera publicación que abarca la inclinación productiva de estos OPC en específico. Aspectos que estarán en función de contribuir a consumar una definición del estado racial de las Sudán, lo que a su vez incentivará a los productores a la cría y mejora de dichos OPC, al igual que el interés de diferentes partes en realizar más investigaciones en torno a estos, conllevando ello finalmente al fomento y conservación de este recurso zoogenético, el cual se ve amenazado por la hibridación sin control de su material genético con razas extranjeras. Se sugiere que se realicen futuros estudios en cuanto a la morfología con una mayor muestra poblacional, para corroborar y complementar los resultados obtenidos.

\section{Conflicto de intereses}

Los autores del presente estudio declaramos que no existe conflicto de intereses con la publicación de este manuscrito.

\section{Agradecimientos}

Agradecimientos a la Universidad de Córdoba por el apoyo económico proporcionado, a la Asociación de Criadores de Ganado Ovino de Colombia (ASOOVINOS) por la mediación con los productores, al igual que a Lina Violet, Leidy Bedoya y Lesly Cabrias por la ayuda brindada en la recolección de datos.

\section{REFERENCIAS}

1. Delgado JV, Perez Grovas R, Camacho ME, Fresno M, Barba C. The Wool-less Canary sheep and their relationship with the present breeds in America. Anim Genet Resour. 2000; 28:27-34. https://doi.org/10.1017/ $\underline{\mathrm{S} 1014233900001334}$

2. Vivas Ascue NJ. Diversidad Genética de Ovinos Criollos Colombianos. [Tesis M.Sc.]. Palmira: Universidad Nacional de Colombia Sede; 2013. URL Disponible en: http:// bdigital.unal.edu.co/12683/
3. Flórez Murillo JM, Hernández Pérez M de J, Bustamante Yánez M de J, Vergara Garay OD. Caracterización morfoestructural de tres poblaciones de Ovino de Pelo Criollo Colombiano "OPC". Arch Zootec. 2018; 67(259):340-348. https://doi. org/10.21071/az.v67i259.3789

4. Arredondo BJV, Rivera DF, Hernández HDY. Tipología morfoestructural de la hembra ovina de pelo criollo colombiano en Quindío y Valle del Cauca. REDVET. 2017; 18(12):1-15. 
5. Ocampo RJ, Martínez RA, Rocha JF, Cardona $\mathrm{H}$. Genetic characterization of Colombian indigenous sheep. Rev Colomb Cienc Pecu. 2017; 30(2):116-125. https:// revistas.udea.edu.co/index.php/rccp/ article/view/325659

6. Ángel S, Ramírez A. Estudio zoométrico del ovino de pelo criollo colombiano de la zona norte del departamento del Huila (Colombia). AICA 2014; 4:338-340. http://www.uco.es/ conbiand/aica/templatemo 110 lin photo/ articulos/2014/Trabajo058 AICA2014.pdf

7. Moreno MJ, Montes VD, Ucrós PJ, Fernández QA, Cardona ÁJ. Variabilidad morfoestructural de la hembra ovina de pelo criollo colombiana. Livestock Res Rural Dev. 2013; 25(5). http://www.Irrd.cipav.org.co/ Irrd25/5/more25083.htm

8. Yunusa AJ, Salako AE, Oladejo OA. Morphometric characterization of Nigerian indigenous sheep using multifactorial discriminant analysis. IJBC 2013; 5(10):661665. http://citeseerx.ist.psu.edu/viewdoc/ download?doi=10.1.1.876.2246\&rep=rep1 $\underline{\text { \&type }}=\mathrm{pdf}$

9. Popoola MA. Zootechnical Index Analysis of West African Dwarf Rams in Southwestern Nigeria. ATS 2015; 48(1-2):24-29. https:// doi.org/10.1515/ats-2015-0004

10. Morales-delaNuez A, Rodríguez C, Santana M, Hernandez-Castellano LE, Niño T, Reyes-Chacon RJ, et al. Morphological measurements and indexes as a tool to support molecular genetic studies: an example in Canary Islands. J Appl Anim Res. 2012; 40(3):215-221. https://doi.org/10.1 $\underline{080 / 09712119.2012 .658062}$

11. Bravo S, Sepúlveda N. Índices zoométricos en ovejas criollas Araucanas. Int J Morphol. $2010 ; 28(2): 489-495$. http://dx.doi. org/10.4067/S0717-95022010000200025

12. Di Rienzo JA, Casanoves F, Balzarini MG, Gonzalez L, Tablada M, Robledo YC. InfoStat. Versión 2016. Argentina: Universidad Nacional de Córdoba; 2016.

13. Arredondo V, Macedo R, Magaña JC, Molina J, Prado O, García LJ, et al. Variabilidad morfológica de la oveja Pelibuey en Colima, México. AICA 2013; 3:1-7. URL Disponible en: http://www.uco.es/conbiand/aica/ templatemo 110 lin photo/articulos/2013/ Trabajo001 AICA2013.pdf
14. Herrera M, Luque M. Morfoestructura y Sistemas para el Futuro en la Valoración Morfológica. En: Sañudo C dir. Valoración Morfológica de los Animales Domésticos. Madrid: Ministerio de Medio Ambiente y Medio Rural y Marino; 2009. https:// www.mapa.gob.es/es/ganaderia/temas/ zootecnia/LIBRO \% 20valoracion \% 20 morfologica\%20SEZ tcm30-119157.pdf

15. Biagiotti $D$, Rocha $S J L$, Oliveira do ÓA, Rêgo NA, Vieira G, da Silva NS, et al. Caracterização fenotípica de ovinos da raça Santa Inês no Estado do Piauí. Rev Bras Saúde Prod Anim. 2013; 14(1):2942. http://dx.doi.org/10.1590/S1519$\underline{99402013000100004}$

16. Vilaboa AJ, Bozzi R, Díaz RP, Bazzi L. Conformación corporal de las razas ovinas Pelibuey, Dorper y Katahdin en el estado de Veracruz, México. Zootecnia Trop. 2010; 28(3):321-328. http://www.bioline.org.br/ pdf?zt10032

17. León AL. Variables morfométricas del ovino Pelibuey Cubano adulto. Rev Prod Anim. 2008; 20(1):72-75. https://revistas.reduc. edu.cu/index.php/rpa/article/view/3017

18. Álvarez S, Fresno M, Capote J, Delgado J, Barba C. Estudio para la caracterización de la raza ovina Canaria. Arch Zootec. 2000; 49(185):209-215. https://www.redalyc. org/articulo.oa?id $=49518630$

19. Dzib CA, Ortiz A, Torres HG. Variabilidad morfoestructural de ovinos Blackbelly en Campeche, México. Arch Zootec. 2011; 60(232):1291-1301. http://dx.doi. org/10.4321/S0004-05922011000400044

20. De la Rosa SA, Revidatti MA, Tejerina ER, Orga A, Cappello JS, Petrina JF. Estudio para la caracterización de la oveja criolla en la región semiárida de Formosa, Argentina. AICA. 2012; 2(1):87-94. http://www. uco.es/conbiand/aica/templatemo 110 lin photo/articulos/2012/Trabajo041 AICA2012.pdf 